\title{
Paracoccidioidomicosis en cavidad oral
}

\section{Oral cavity paracoccidioidomycosis}

\author{
Antunes Freitas D*, Vergara Hernández CI**, Díaz Caballero A***, Moreira G****
}

\section{RESUMEN}

La paracoccidioidomicosis (PCM) o blastomicosis suramericana es la micosis sistémica más importante de América latina que es relativamente común en Brasil, Venezuela, Colombia, Ecuador y Argentina. Los casos esporádicos también pueden verse en algunos otros países, la cual es progresiva y con un infrecuente desenlace fatal si no es tratada a tiempo. Se considera como una enfermedad multifocal, con lesiones orales como la característica prominente. Es causada por un hongo dimórfico, Paracoccidioides brasiliensis, que afecta principalmente la piel, los ganglios linfáticos, los pulmones y membranas mucosas oral, nasal y gastrointestinal. Dependiendo de la inmunidad específica del huésped, la infección puede asumir muchas formas y afecta a uno o varios órganos, llegando a ser una enfermedad grave y potencialmente fatal. Es muy importante para los profesionales de la salud de todo el mundo tener conocimiento acerca de la Paracoccidioidomicosis porque a veces la enfermedad sólo se manifiesta muchos años después de que haya abandonado la zona endémica. Para proporcionar información útil sobre el diagnóstico y tratamiento de la enfermedad se presenta caso clínico de un paciente masculino de 48 años de edad procedente de una zona rural de Juramento Brasil, por presentar múltiples úlceras dolorosas en encía y paladar de 3 meses de evolución; refiere antecedentes de fumador crónico, al examen clínico extraoral se descartan lesiones en otros órganos y al examen intraoral se observan múltiples úlceras con fondo necrótico y granulomatoso localizadas en encía y paladar. Se realizó una biopsia incisional de la lesión y el material fue enviado para estudio anatomopatológico. El informe histopatológico confirmó la impresión clínica de Paracoccidioidomicosis. El paciente fue tratado con el uso de sulfametoxazol + trimetoprima - 800/60 mg/día, vía oral, cada 12 horas durante 30 días. Las lesiones bucales desaparecieron completamente y el paciente continúa bajo control en el servicio.

Palabras clave: Paracoccidioidomicosis, blastomicosis suramericana, enfermedad micótica.

\section{SUMMARY}

Paracoccidioidomycosis (PCM) or South-american Blastomycosis is the most important systemic mycosis in Latin America, is relatively common in Brazil, Venezuela, Colombia, Ecuador and Argentina. Sporadic cases can also be seen in some other countries, which is a rare progressive and fatal if not treated. It has been regarded as a multifocal disease with oral lesions as the prominent feature. It is caused by a dimorphic fungus, Paracoccidioides brasiliensis, which mainly affects the skin, lymph nodes, lungs and mucous membranes, oral, nasal and gastrointestinal. Depending on the specific immunity of the host, the infection can take many forms

* Odontólogo UNICOR. Especialista en Bioética UFLA. Maestría en Diagnóstico y Patología UNICOR. Profesor titular Facultad de Odontología FUNORTE Brasil, Campus da Saúde.

** Odontóloga Universidad de Cartagena. Especialista en Patología y Cirugía Oral Universidad Javeriana. Profesor titular, Vicedecana Curricular de la facultad de Odontología de la universidad de Cartagena Facultad de Odontología Universidad de Cartagena.

*** Odontólogo Universidad de Cartagena. Periodoncista Universidad Javeriana. Profesor titular U de C. Facultad de Odontología Universidad de Cartagena.

**** Odontóloga UNIMONTES. Maestría Patología Oral UFMG. Profesor titular Facultad de Odontología FUNORTE Brasil, Campus da Saúde. 
and affects one or more organs, becoming a serious and potentially fatal disease. It is very important for health professionals worldwide have knowledge about Paracoccidioidomycosis because sometimes the disease only manifests itself many years after you have left the endemic area. To provide useful information about the diagnosis and treatment of disease A case report of case of male patient 48 years old from a rural area of Brazil Oath, presenting multiple painful ulcers on the gums and palate of 3 months duration, referred to chronic smoking history, clinical examination extraoral lesions are discarded in other intraoral examination bodies and multiple ulcers were observed necrotic and granulomatous background located on the gums and palate. We performed an incisional biopsy of the lesion and the material was sent for pathologic examination. The pathology report confirmed the clinical impression of Paracoccidioidomycosis. The patient was treated with the use of sulfamethoxazole + trimethoprim - 800/60 mg/day, orally every 12 hours for 30 days. The oral lesions disappeared completely and the patient remains under control in the service.

Key words: Paracoccidioidomycosis, south-american blastomycosis, mycotic disease (Mesh Database)..

Fecha de recepción: 1 de noviembre de 2010.

Aceptado para publicación: 15 de noviembre de 2010.

Antunes Freitas D, Vergara Hernández CI, Díaz Caballero A, Moreira G. Paracoccidioidomicosis en cavidad oral. Av. Odontoestomatol 2012; 28 (1): 11-16.

\section{INTRODUCCIÓN}

La paracoccidioidomicosis es una enfermedad micótica causada por el brasiliensis Paracoccidioides, común en los suelos de cultivo y cría de ganado que se observa más comúnmente en pacientes que viven en América del Sur o Central que se encuentra en focos endémicos en la zona que se extiende entre México por el norte hasta Argentina por el sur, con la mayoría de los casos en Brasil, Venezuela y Colombia.

Esta enfermedad se caracteriza porque, al igual que otras micosis profundas como la histoplasmosis y la coccidioidomicosis, presenta afección pulmonar primaria y se disemina desde el foco inicial para afectar otros órganos, ya que su diseminación es por vía hematógena. El contagio se lleva a cabo por la aspiración de tierra contaminada o por vía transcutánea en los individuos particularmente susceptibles.

Su forma más común de infección es por inhalación de esporas, es endémica en la zona de los Andes Venezolanos y de Colombia, especialmente en zonas de cultivo de café y también en ciertas zonas de Brasil, como Minas Gerais (1-3).

\section{CARACTERÍSTICAS CLÍNICAS}

Son raros los casos reportados en cavidad bucal, algunos asociados con Carcinoma escamocelular, sin embargo, en esta infección, los signos iniciales son generalmente pulmonar (del pulmón debido a la participación), incluyendo tos, fiebre, sudores nocturnos, pérdida de peso, dolor en el pecho, adenopatías y hemoptisis $(1,4,5)$. Las lesiones bucales se presentan en forma de úlceras crónicas dolorosas que no sanan espontáneamente. Estas úlceras tienen la característica típica de una superficie granulomatosa purpúrea. El sitio de aparición más frecuente para éstas lesiones son las encías, pero pueden observarse también en el paladar, lengua y el resto de la mucosa medida que evolucionan, las úlceras adquieren un aspecto "de mora" que da a las lesiones un puntillazo característico. En etapas avanzadas de la enfermedad, además del compromiso sistémico del paciente por la infección pulmonar, se puede observar destrucción ósea progresiva de los maxilares afectados, lo que da lugar a una recesión gingival con exposición de las raíces dentarias y pérdida dentaria. La mucosa gingival adquiere una consistencia blanda, eritematosa y edematosa. También puede haber un compromiso de los tractos digestivo y respiratorio superiores a bucal (3, 6-9). 
Se presenta más en hombres de 30 a 60 años, de oficios relacionados con la tierra $(1,3)$. Las lesiones oro faciales causadas por micosis sistémicas han sido poco reportadas en el pasado en pacientes que trabajan en áreas geográficas con una alta prevalencia de infección y ocasionalmente en individuos inmunocomprometidos. El incremento del turismo y la facilidad de viajar hasta cualquier área del mundo y el dramático incremento de los pacientes con el Virus de la Inmunodeficiencia Humana (VIH), son los mayores responsables de un aumento en los reportes de lesiones oro faciales de micosis sistémicas y actualmente se reconocen nuevas enfermedades oportunistas. Los profesionales de la salud bucal y bucofacial deben ahora tomar en cuenta la posibilidad de la presencia de una enfermedad micótica sistémica al observar ulceraciones crónicas, infección crónica de los senos maxilares, o lesiones bucales idiopáticas e inusuales, sobre todo en pacientes $\mathrm{VIH}+$, con desórdenes linfoproliferativos o diabéticos, o también en aquellos que viven en áreas endémicas. El diagnóstico y manejo clínico de los pacientes con lesiones micóticas sistémicas debe ser hecho multidisciplinariamente ya que pueden estar involucrados los pulmones y otros órganos importantes además de la cavidad bucal (10).

\section{CARACTERÍSTICAS HISTOPATOLÓGICAS}

La forma de levadura del Paracoccidioides brasiliensis, el hongo causal, es de gran tamaño (1060 micras) y muestra características de gemación múltiple en torno a una célula madre, dando lugar a la formación de una roseta fúngica o "imagen en rueda de timón". En cultivos P. brasiliensis se ve como crecimiento de esporas grandes de doble contorno que muestran múltiples brotes $(5,11)$.

\section{DIAGNÓsTICO}

El diagnóstico clínico se hace basado en la anamnesis y el aspecto clínico de las lesiones, este diagnóstico debe confirmarse realizando cultivo y frotis de las lesiones y biopsia incisional. Se comprueba la presencia del hongo mediante la prueba de la paracoccidioidina (intradérmica) y serología. Para confirmar si se involucran las vías aéreas (como suele su- ceder) se realiza Rx de tórax y si se sospecha la afectación de vías digestivas se procede a realizar una endoscopia gástrica con toma de cultivo y/o biopsia incisional. Se ha descrito el uso de la técnica de hibridación in situ para incrementar la eficacia del diagnóstico de paracoccidioidomicosis bucal $(7,12-14)$.

\section{TRATAMIENTO}

El tratamiento de las micosis profundas y/o sistémicas se hace desde 1978 con Anfotericina B y/o Flucitosina. Los imidazoles: miconazol y ketoconazol, fueron introducidos durante los siguientes tres años. Si se administra intravenosamente, el miconazol sirve como agente terapéutico limitante, pero no tiene una vida media activa prolongada en sangre. El ketoconazol administrado vía oral es una opción efectiva, no costosa y conveniente para tratar candidiasis mucosa, y fue ampliamente utilizada durante toda una década ya que no existía ningún otro agente antimicótico efectivo para tratar micosis sistémicas profundas. Durante la década de los años noventa el uso del ketoconazol disminuyó debido a la aparición de los triazoles: fluconazol e imidazol. El fluconazol es menos tóxico y tiene algunas ventajas farmacológicas sobre el ketoconazol, como la penetración dentro del líquido cerebroespinal; además tiene una eficacia mayor en contra de candidiasis, criptococosis y coccidioidomicosis sistémicas. El Itraconazol es un antimicótico derivado de los triazoles de espectro muy amplio. Cuando se administra oralmente, el Itraconazol alcanza altos niveles de concentración y los mantiene en muchos tejidos, como la piel, las uñas y la mayoría de los órganos profundos. Su valor clínico ha sido demostrado en todas las formas de micosis que afectan piel y mucosas y en las onicomicosis. Es la droga de elección para infecciones sistémicas como blastomicosis, histoplasmosis, paracoccidioidomicosis, esporotricosis linfocutánea, aspergilosis crónica diseminada y muchos casos de candidiasis y criptococosis profundas y de larga data de evolución, aún cuando la infección micótica ya alcanza las meninges u otro órgano del SNC $(2,10)$.

El itraconazol posee una excelente absorción en el estómago y sus niveles en plasma sanguíneo después de administrar oralmente $100 \mathrm{mg}$ es de 0,16 microgr/ml a las 3-4 horas después de la ingestión 
de la droga. La vida media del Itraconazol va de 17 a 21 horas y el 99,8\% de la droga se une a proteínas plasmáticas, especialmente la albúmina. Se metaboliza a través del hígado, donde se forman metabolitos inactivos, a excepción del hidroxi-itraconazol, el cual exhibe una actividad antimicótica discreta. En la piel y particularmente en las uñas, el itraconazol persiste por largo tiempo después de descontinuar la terapia. Su mecanismo de acción es similar al de otros azoles, inhibiendo la alfa-14-dimetilasa de ianosterol, la cual interfiere con la síntesis de ergosterol (uno de los compuestos de la pared celular del hongo). El itraconazol no actúa en contra de los Zigomicetos. Las dosis diarias varían de 100 a $400 \mathrm{mg}$. La anfotericina sigue siendo la terapia estándar de elección para las micosis más profundas, mientras que los nuevos azoles son los agentes de primera línea para las micosis superficiales como la candidiasis, su uso está actualmente incrementándose en las micosis profundas Actualmente está en desarrollo un nuevo medicamento, el SPZ o saperconazole, con el que se reportan excelentes resultados en el tratamiento de micosis sistémicas y subcutáneas $(5,6)$.

\section{PRESENTACIÓN DEL CASO}

Paciente de 48 años de edad, género masculino, caucásico, procedente de Juramento Minas Gerais, asistió a la Clínica de Diagnóstico Bucal do Campus de Saúde de la Funorte-Brasil remitido por un odontólogo cirujano de la ciudad. En la entrevista, el paciente informó que hace tres meses, aparecieron múltiples úlceras dolorosas en la boca. El paciente no reportó cambios sistémicos adicionales. El paciente refirió ser residente de la zona rural de su ciudad, que hace uso de cigarrillos muy a menudo, con una duración de más de 20 años en este hábito. También hace uso de bebidas alcohólicas con regularidad. Al examen físico extraoral no se produjeron cambios significativos. Al Examen clínico de cavidad oral se detecta la presencia de úlceras intraorales, las cuales se observaron en el paladar y encías con apariencias de úlceras granulomatosas de fondo eritematoso. Se realizó una biopsia incisional de la lesión y el material fue enviado para estudio anatomopatológico. El informe histopatológico confirmó la impresión clínica de Paracoccidioidomicosis. El ma-

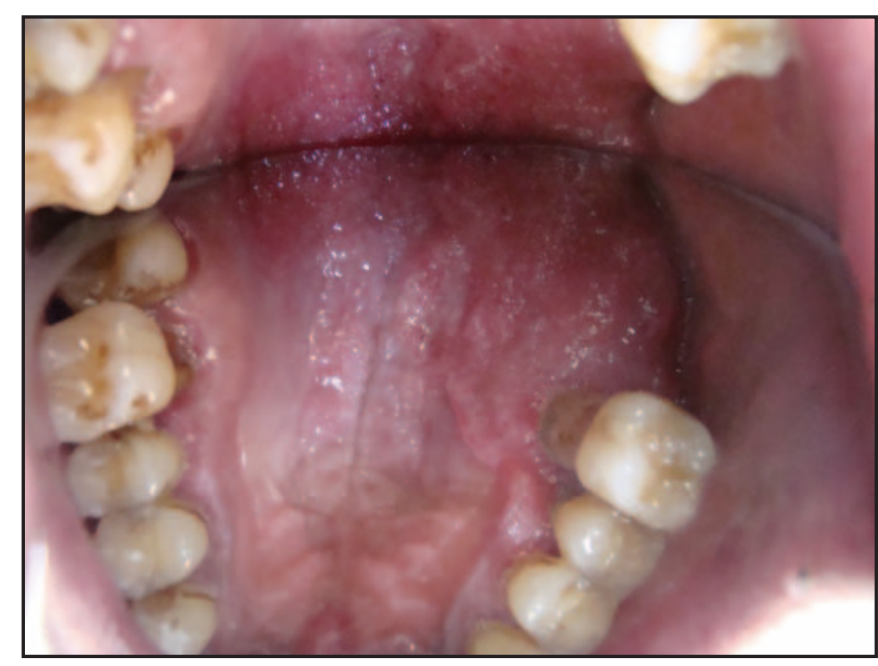

Fig. 1. Se observan lesiones en cavidad oral del paciente.

terial de la hoja manchada demostró infiltrado inflamatorio y granulomatoso con células gigantes multinucleadas y la presencia de elementos fúngicos compatibles con $P$. brasiliensis. El paciente fue derivado a un médico especialista en enfermedades infecciosas y los exámenes médicos mostraron que la lesión se encontraba sólo en la cavidad oral. El paciente fue tratado con el uso de sulfametoxazol + trimetoprim $800 / 60 \mathrm{mg} /$ día, vía oral, cada 12 horas durante 30 días. Las lesiones bucales desaparecieron completamente y el paciente está bajo seguimiento clínico en el servicio.

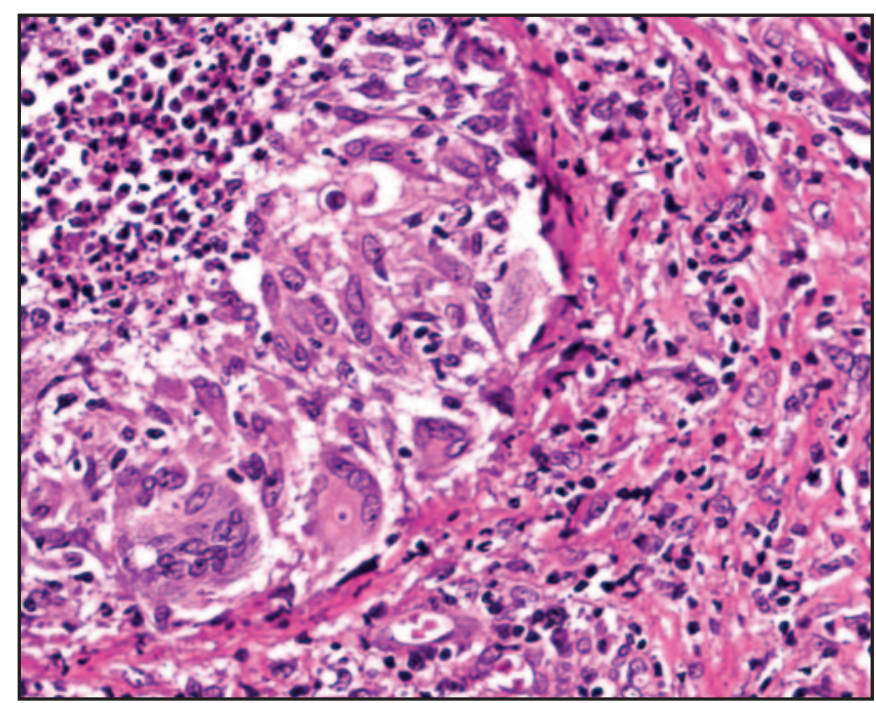

Fig. 2. Imagen histológica de la muestra obtenida de la biopsia del paciente. 


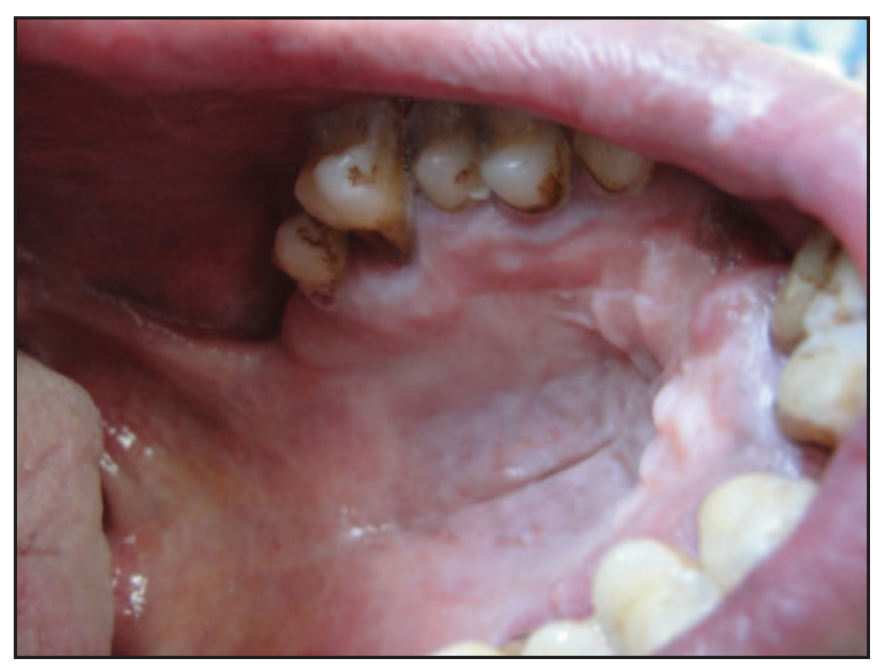

Fig. 3. Se observa el grado de resolución de la patología, posterior al tratamiento.

\section{DISCUSIÓN}

La prevalencia de infecciones micóticas en países latinoamericanos no es tan elevada de acuerdo a lo reportado en diversos artículos relacionados con el tema de las micosis profundas o sistémicas. Para ser considerada una zona geográfica como endémica para infecciones micóticas, se deben tener en cuenta al menos 3 factores importantes, tales como pueden ser a) prevalencia de infección superior al 5\%, b) aislar por medio de laboratorio el agente causal del ambiente y c) la detección de casos clínicos de estas micosis (1). La presencia de paracoccidioidomicosis en la zona de residencia del paciente no tiene reportada de forma estructurada un OR que permita establecer que se pueda considerar como una patología endémica típica de alguna zona determinada.

La valoración de los pacientes en zonas no urbanas, con patologías en cavidad oral con apariencias clínicas vegetantes, exfoliativas, de larga duración y de uso de diversas terapéuticas sin un éxito adecuado, de forma inmediata debe abrir la opción del uso de la biopsia como una forma científica y certera de poder llegar a un diagnóstico definitivo que le facilite a los pacientes la solución de sus patologías, con un enfoque mucho más científico y menos empírico $(12,13)$. En el paciente que se reporta, la importancia de la aplicación de la biopsia como estrategia de diagnóstico, fue de manera categórica y fundamental para el establecimiento de diagnóstico certero que permite iniciar un tratamiento farmacológico adecuado.

La interacción en diferentes niveles de complejidad desde el odontólogo general, hasta el odontólogo especialista en patología oral y en cirugía, para posteriormente ser tratado por el médico especialista en infecciones, permite establecer de forma certera que la complementación de cada uno de los profesionales tratantes y con diferentes niveles de complejidad, más que mirarse como la oportunidad de ser excluyentes en los tratamiento, debe permitir la oportunidad de ver como se complementan en su nivel, cada una de las profesiones tratantes y lo oportuno de la acción interprofesional. En el presente caso el beneficio de ese enfoque le produjo un resultado clínico grato y de excelente resultado, lo que se traduce en la recuperación de la salud, la prevención de las complicaciones orales o sistémicas y en el seguimiento, para prevenir la recidiva temprana de la patología tratada.

De las diferentes opciones de tratamiento, la elección del tratamiento del paciente reportado en el artículo, muestra que la farmacología con un diagnóstico certero por medio del análisis histopatológico, se reflejará en la resolución temprana de la patología a la cual se está enfrentado. Es notorio que los resultados clínicos y microbiológicos del paciente tratado, demuestran que la elección de un enfoque terapéutico tipo antibiótico, no debe ser empírico, sino por lo contrario, llenarse de razones biomédicas para lograr un resultado excelente.

\section{BIBLIOGRAFÍA}

1. Brazao-Silva MT, Andrade MF, Franco T, Ribeiro RI, Silva WD, Faria G, et al. Paracoccidioidomycosis: a series of 66 patients with oral lesions from an endemic area. Mycoses. Apr 6.

2. Marques SA, Camargo RM, Abbade LP, Fortaleza CM, Marques ME. Paracoccidioidomycosis: an unusual presentation in a young girl disclosing an unnoted HIV-infection. Med Mycol. Feb;48(1): 182-7.

3. Ramos ESM, Saraiva Ldo E. Paracoccidioidomycosis. Dermatol Clin. 2008 Apr;26(2):257-69, vii. 
4. Meneses-Garcia A, Mosqueda-Taylor A, Moralesde la Luz R, Rivera LM. Paracoccidioidomycosis: report of 2 cases mimicking squamous cell carcinoma. Oral Surg Oral Med Oral Pathol Oral Radiol Endod. 2002 Nov;94(5):609-13.

5. Andrade MG, Medrado AP, de Brito IC, de Almeida Reis SR. Oral paracoccidioidomycosis: a case without lung manifestations. J Contemp Dent Pract. 2007;8(5):92-8.

6. Godoy H, Reichart PA. Oral manifestations of paracoccidioidomycosis. Report of 21 cases from Argentina. Mycoses. 2003;46(9-10):412-7.

7. Kaminagakura E, Graner E, de Almeida OP, Di Hipolito Junior O, Lopes MA. Oral paracoccidioidomycosis or squamous cell carcinoma? Gen Dent. 2004 Jan-Feb;52(1):48-50.

8. Bicalho RN, Santo MF, de Aguiar MC, Santos VR. Oral paracoccidioidomycosis: a retrospective study of 62 Brazilian patients. Oral Dis. 2001 Jan; 7(1):56-60.

9. Almeida OP, Jacks J Jr, Scully C. Paracoccidioidomycosis of the mouth: an emerging deep mycosis. Crit Rev Oral Biol Med. 2003;14 (5):377-83.

10. Paniago AM, de Freitas AC, Aguiar ES, Aguiar JI, da Cunha RV, Castro AR, et al. Paracoccidioido- mycosis in patients with human immunodeficiency virus: review of 12 cases observed in an endemic region in Brazil. J Infect. 2005 Oct;51(3):248-52.

11. Silva CO, Almeida AS, Pereira AA, Sallum AW, Hanemann JA, Tatakis DN. Gingival involvement in oral paracoccidioidomycosis. J Periodontol. 2007 Jul;78(7):1229-34.

12. Talhari C, de Souza JV, Parreira VJ, Reinel D, Talhari S. Oral exfoliative cytology as a rapid diagnostic tool for paracoccidioidomycosis. Mycoses. 2008 Mar;51(2):177-8.

13. Torres-Pereira C, Giovanini AF, Stramandinoli RT, Amenabar JM, Piazzetta CM. Oral paracoccidioidomycosis and pulmonary tuberculosis co-infection: relevance of oral biopsy in establishing the diagnosis and therapeutic approach. Int J Infect Dis. 2009 Jan;13(1):114-6.

14. Kaminagakura E, Bonan PR, Lopes MA, Almeida OP. Cell proliferation and p53 expression in pseudoepitheliomatous hyperplasia of oral paracoccidioidomycosis. Mycoses. 2006 Sep;49 (5):393-6.

\section{CORRESPONDENCIA}

Clara Inés Vergara Hernández

Correo electrónico: cvergarahdez@gmail.com 